\title{
ACTIVE MOSS BIOMONITORING OF TRACE ELEMENTS AIR POLLUTION IN CHISINAU, REPUBLIC OF MOLDOVA
}

\author{
BIOMONITORING AKTYWNY \\ Z WYKORZYSTANIEM MCHÓW, PIERWIASTKÓW ŚLADOWYCH \\ W POWIETRZU W MIEŚCIE CHISINAU, REPUBLIKA MOŁDAWII
}

\begin{abstract}
For the first time active moss biomonitoring was used to assess trace element deposition in the capital of the Republic of Moldova, Chisinau. Moss Sphagnum girgensohnii samples were exposed in bags at three sites of Chisinau from October, 2016 to March, 2017. The content of 30 elements: $\mathrm{Na}, \mathrm{Mg}, \mathrm{Cl}, \mathrm{K}, \mathrm{Ca}, \mathrm{Sc}, \mathrm{Ti}, \mathrm{V}, \mathrm{Cr}$, $\mathrm{Mn}, \mathrm{Fe}, \mathrm{Co}, \mathrm{Ni}, \mathrm{Zn}, \mathrm{As}, \mathrm{Br}, \mathrm{Rb}, \mathrm{Mo}, \mathrm{Sr}, \mathrm{Sb}, \mathrm{Ba}, \mathrm{La}, \mathrm{Ce}, \mathrm{Cs}, \mathrm{Hf}, \mathrm{Th}, \mathrm{Cu}, \mathrm{Cd}, \mathrm{Pb}$, and $\mathrm{U}$ in the exposed and unexposed mosses was determined by neutron activation analysis and atomic absorption spectrometry. According to the relative accumulation factor, the most abundant elements in the samples were $\mathrm{V}, \mathrm{Cr}, \mathrm{Fe}, \mathrm{Ba}, \mathrm{La}, \mathrm{As}, \mathrm{Sb}, \mathrm{U}$, and $\mathrm{Pb}$. Such elements as $\mathrm{Cl}, \mathrm{K}$, and $\mathrm{Rb}$ were depleted from the moss tissue during the time of exposure. Principal component analysis was used to identify and characterize different pollution sources. The obtained results indicate that the use of $S$. girgensohnii moss bags is a simple and inexpensive technique to monitor major and trace element content in the air of urban area.
\end{abstract}

Keywords: active moss biomonitoring, Sphagnum girgensohnii, elemental content, urban area, neutron activation analysis, atomic absorption spectrometry

\section{Introduction}

More than 40 years the moss transplant technique introduced by Goodman and Roberts in 1971 has been widely used to monitor air quality in industrial [1-3] as well as in urban areas $[4,5]$. Moss bag technique allowed overcoming the problem of the lack of native moss in areas affected by relatively high pollutant levels or unsuitable climatic and environmental conditions. This technique has many advantages: well-defined exposure time; known concentration of elements in the unexposed sample, the uniformity of entrapment surface and flexibility both in site selection and in number of sites that can be chosen [4, 6]. Despite the wide use of moss bag technique, there is still not a well-established protocol for the use of moss as monitoring tool in urban areas $[5,7]$.

\footnotetext{
${ }^{1}$ Joint Institute for Nuclear Research, 6 Joliot-Curie Str., 1419890, Dubna, Russia

${ }^{2}$ Horia Hulubei National Institute for R\&D in Physics and Nuclear Engineering, 30 Reactorului Str. MG-6, Bucharest - Magurele, Romania

${ }^{3}$ The Institute of Chemistry of the ASM, 3 Academiei Str., 2028 Chisinau, Moldova

${ }^{4}$ Institute of Physics Belgrade, University of Belgrade, Pregrevica 118, Belgrade 11080, Serbia

*Corresponding author: zinikovskaia@mail.ru
} 
Sphagnum species have been considered especially suitable for the monitoring of heavy metal pollution due to their high cation-exchange capacity of cell membrane, large area/volume ratio, high permeability of tissues to water and elements and high water retention capacity $[4,7]$.

Currently, air pollution is one of the most serious problems in the Republic of Moldova and particularly in Chisinau. The quality of air in the Chisinau is determined by two main pollution sources: mobile (vehicles) and stationary (the power and heat generation sector, as well as industry) [8]. According to the national reports, in air samples mainly the concentrations of particulates, sulphur dioxide, carbon monoxide, nitrogen dioxide, phenols, and formaldehyde are determined [9]. At the same time, information concerning determination of heavy metals is very limited. Begu [10] used lichens to monitor the ecological situation in Chisinau during 9-month period. The concentration of six elements $(\mathrm{Cu}, \mathrm{Pb}, \mathrm{Zn}, \mathrm{Cd}, \mathrm{Cr}$, and $\mathrm{Ni})$ determined by atomic absorption spectrometer showed their significant accumulation during the exposure time of the lichen samples.

In the present study, samples of Sphagnum girgensohnii were exposed in bags at three different sites (Thermal power plant, Academy of Science main building and Botanical garden) of Chisinau, Republic of Moldova from October 2016 to March 2017 to examine the ecological situation in an urban area during heating season. Combination of two high sensitive analytical techniques: neutron activation analysis (NAA) and atomic absorption spectrometry (AAS) allowed determination of the inorganic pollutants previously not determined in Chisinau.

\section{Experimental}

Study area. The study was carried out in the city of Chisinau, the capital of Republic of Moldova, the main industrial centre of the country and its largest transportation hub. The climate in Chisinau is humid continental transitional with a subtropical, characterized by hot dry summers and windy cold winters.

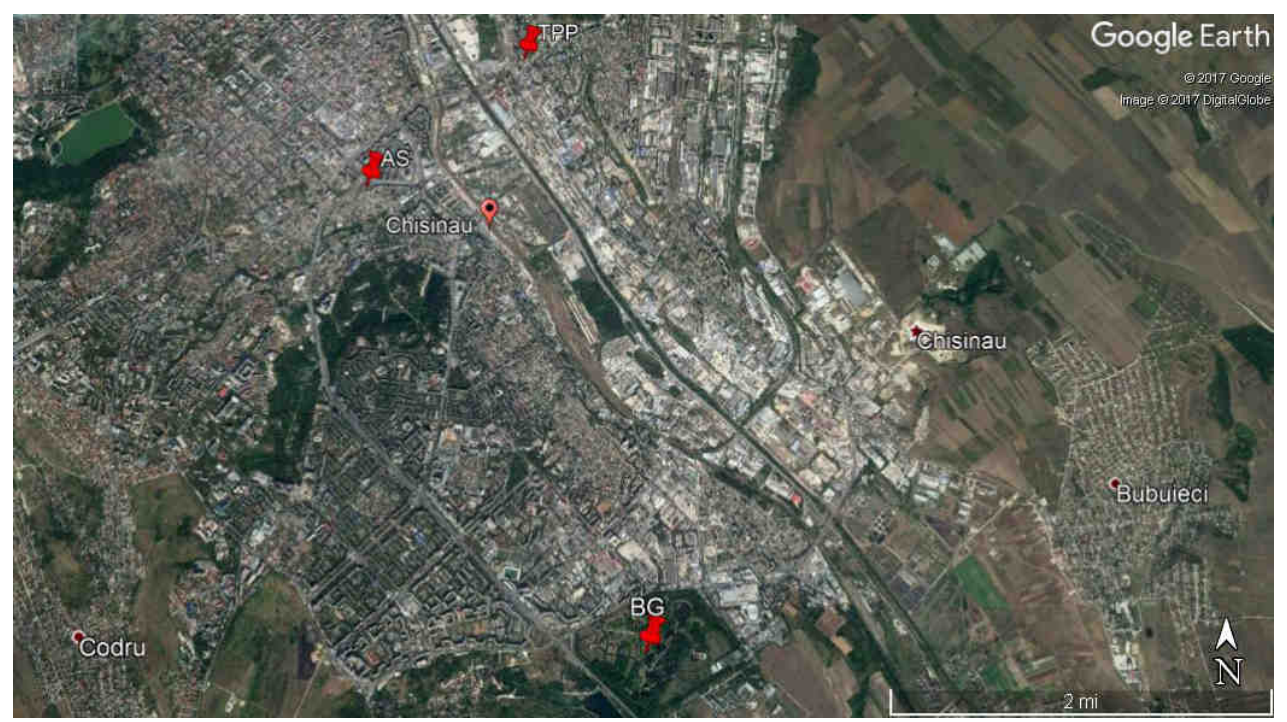

Fig. 1. Exposure sites of the $S$. girgensohnii moss bags: TPP, BG and AS 
Moss Sphagnum girgensohnii Russow (S. girgensohnii) was collected in 2016 from a pristine territory located in Tver region, Russian Federation. Moss samples for exposure were prepared according to procedure described in Anicic et al. [4] study. Moss bags were exposed at three different sites: the thermal power plant (TPP) and Academy of Science main building (AS), potentially polluted places and in Botanical garden (BG) considered as an unpolluted site (Fig. 1). Moss samples were not washed before exposure. As it was shown in the Anicic et al. [11] study, the difference in the elemental concentrations of $S$. girgensohnii in washed and unwashed samples was insignificant. At each site, fifteen bags were suspended from October, 2016 to March, 2017 (the period considered heating season in Chisinau). Every month three of them were collected and kept in tightly closed paper and plastic bags. A part of the unexposed moss material was kept in the laboratory and further used as a reference sample. Sample preparation for NAA and AAS analyses was done in accordance with the methodology described by Zinicovscaia et al. [12].

Methods. Neutron activation analysis was carried out at the pulsed fast reactor IBR-2 of the Frank Laboratory of Neutron Physics, JINR, Dubna, Russia. A total of 27 elements ( $\mathrm{Na}, \mathrm{Mg}, \mathrm{Cl}, \mathrm{K}, \mathrm{Ca}, \mathrm{Sc}, \mathrm{Ti}, \mathrm{V}, \mathrm{Cr}, \mathrm{Mn}, \mathrm{Fe}, \mathrm{Co}, \mathrm{Ni}, \mathrm{Zn}, \mathrm{As}, \mathrm{Br}, \mathrm{Rb}, \mathrm{Mo}, \mathrm{Sr}, \mathrm{Sb}, \mathrm{Ba}, \mathrm{La}, \mathrm{Ce}$, Hf, Th and U) were determined in the moss samples. The concentrations of elements based on short-lived radionuclides: $\mathrm{Cl}, \mathrm{V}, \mathrm{Mg}, \mathrm{Ca}, \mathrm{Ti}$ and $\mathrm{Mn}$ were determined by irradiation for $3 \mathrm{~min}$ at a thermal neutron flux of $1.6 \times 1013 \mathrm{n} \mathrm{cm}^{-2} \mathrm{~s}^{-1}$. To determine long-lived isotopes of $\mathrm{Na}, \mathrm{Sc}, \mathrm{Cr}, \mathrm{Fe}, \mathrm{Co}, \mathrm{Ni}, \mathrm{Zn}, \mathrm{As}, \mathrm{Br}, \mathrm{Rb}, \mathrm{Sr}, \mathrm{Mo}, \mathrm{Sb}, \mathrm{Ba}, \mathrm{La}, \mathrm{Ce}, \mathrm{Hf}$, Th, and U, the samples were irradiated for 3 days at a neutron flux $3.31 \times 1012 \mathrm{n} \mathrm{cm}^{-2} \mathrm{~s}^{-1}$, repacked, and then measured twice after for 4 and 20 days. More details concerning the irradiation and data processing can be found in $[13,14]$. The content of $\mathrm{Cd}, \mathrm{Cu}$, and $\mathrm{Pb}$ in the moss samples was determined by AAS [12].

Quality assurance. To provide quality control of NAA, certified reference materials: SRM 1633c (constituent elements in coal fly ash), SRM 2710 (Montana Soil) and SRM 1547 (peach leaves) were used. In the case of AAS, NIST certified reference materials SRM 1570a (spinach leaves) and SRM 1575 (pine needles) were applied. In both cases, the experimentally measured contents were in a good agreement with the certified values. The difference between certified and measured content of elements of the certified material varied between 3 and $7 \%$ for AAS and between 2 and $15 \%$ for NAA.

Statistical approaches. To assess the element accumulation in the studied moss, relative accumulation factor (RAF) was calculated using the following formula:

$$
R A F=\frac{\left(C_{x}-C_{0}\right)}{C_{0}}
$$

where $C_{0}$ is the initial element concentration, and $C_{x}$ denotes the element concentration in exposed moss.

To highlight any association of the elements as well as to decrease the number of variables for the obtained data, factor analysis (FA) was used. The statistical analysis was performed using Statistica 9 (StatSoft, Tulsa, OK, USA).

\section{Results and discussion}

The results of analysis of the exposed samples and statistical analysis data (including RAF values) are summarized in Tables 1 and 2. As it was shown in Culicov et al. [15], the RAF values calculated for moss samples exposed at different regions cannot be compared because the initial element concentrations in the transplanted moss are different. The initial 
content of elements determined in the moss samples in the present study (Table 3) were in the range or even lower than the data reported for the moss Sphagnum sp. in the other studies $[4,15,16]$.

Elemental content $\left[\mathrm{mg} \mathrm{kg}^{-1}\right.$ d.m.] of the moss exposed for five months (d.m. $=$ dry mass)

Table 1

\begin{tabular}{|c|c|c|c|c|c|}
\hline Site & \multicolumn{5}{|c|}{ TPP } \\
\hline Month & I & II & III & IV & $\mathrm{V}$ \\
\hline $\mathrm{Na}$ & $269 \pm 8$ & $544 \pm 16$ & $608 \pm 32$ & $1370 \pm 82$ & $2720 \pm 163$ \\
\hline $\mathrm{Mg}$ & n.d. & n.d. & n.d. & n.d. & $1040 \pm 62$ \\
\hline $\mathrm{Cl}$ & $394 \pm 35$ & $529 \pm 47$ & $568 \pm 51$ & $2430 \pm 218$ & $1720 \pm 155$ \\
\hline $\mathrm{K}$ & $5020 \pm 400$ & $6890 \pm 550$ & $2440 \pm 190$ & $5020 \pm 400$ & $4440 \pm 350$ \\
\hline $\mathrm{Sc}$ & $0.057 \pm 0.003$ & $0.079 \pm 0.004$ & $0.091 \pm 0.005$ & $0.125 \pm 0.006$ & $0.174 \pm 0.009$ \\
\hline $\mathrm{Ca}$ & $2980 \pm 450$ & $4360 \pm 650$ & $8150 \pm 1220$ & $9680 \pm 1450$ & $6500 \pm 975$ \\
\hline $\mathrm{Ti}$ & $31 \pm 4$ & $72 \pm 10$ & $75 \pm 10$ & $110 \pm 16$ & $53 \pm 8$ \\
\hline $\mathrm{Cr}$ & $0.7 \pm 0.1$ & $2.0 \pm 0.3$ & $1.5 \pm 0.5$ & $2.0 \pm 0.3$ & $2.3 \pm 0.3$ \\
\hline $\mathrm{V}$ & $2.0 \pm 0.1$ & $2.7 \pm 0.1$ & $5.4 \pm 0.3$ & $10.8 \pm 0.5$ & $3.9 \pm 0.2$ \\
\hline $\mathrm{Mn}$ & $239 \pm 12$ & $295 \pm 14$ & $390 \pm 20$ & $327 \pm 16$ & $282 \pm 17$ \\
\hline $\mathrm{Ni}$ & $1.5 \pm 0.2$ & $4.7 \pm 0.7$ & $3.6 \pm 0.5$ & $2.1 \pm 0.3$ & $2.7 \pm 0.4$ \\
\hline $\mathrm{Fe}$ & $276 \pm 12$ & $332 \pm 23$ & $378 \pm 26$ & $514 \pm 35$ & $646 \pm 45$ \\
\hline $\mathrm{Co}$ & $0.25 \pm 0.02$ & $0.29 \pm 0.02$ & $0.33 \pm 0.03$ & $0.41 \pm 0.04$ & $0.47 \pm 0.04$ \\
\hline $\mathrm{Zn}$ & $43 \pm 1$ & $46 \pm 1$ & $88 \pm 3$ & $54 \pm 1$ & $55 \pm 1$ \\
\hline As & $0.74 \pm 0.05$ & $0.76 \pm 0.05$ & $1.04 \pm 0.07$ & $1.20 \pm 0.08$ & $1.24 \pm 0.09$ \\
\hline $\mathrm{Br}$ & $5.9 \pm 0.2$ & $4.9 \pm 0.1$ & $3.5 \pm 0.1$ & $6.4 \pm 0.2$ & $7.1 \pm 0.2$ \\
\hline $\mathrm{Sr}$ & $10 \pm 1$ & $19 \pm 2$ & $24 \pm 2$ & $31 \pm 3$ & $35 \pm 3$ \\
\hline $\mathrm{Rb}$ & $26 \pm 4$ & $31 \pm 5$ & $11 \pm 2$ & $27 \pm 5$ & $19 \pm 3$ \\
\hline Mo & $0.15 \pm 0.04$ & $0.17 \pm 0.05$ & $0.11 \pm 0.03$ & $0.22 \pm 0.07$ & $0.21 \pm 0.06$ \\
\hline $\mathrm{Sb}$ & $0.25 \pm 0.02$ & $0.42 \pm 0.03$ & $0.34 \pm 0.03$ & $0.59 \pm 0.05$ & $1.00 \pm 0.09$ \\
\hline $\mathrm{Ba}$ & $25 \pm 2$ & $18 \pm 2$ & $28 \pm 3$ & $29 \pm 3$ & $29 \pm 3$ \\
\hline $\mathrm{La}$ & $0.21 \pm 0.01$ & $0.28 \pm 0.01$ & $0.28 \pm 0.01$ & $0.48 \pm 0.02$ & $0.57 \pm 0.02$ \\
\hline $\mathrm{Ce}$ & $0.27 \pm 0.03$ & $0.95 \pm 0.09$ & $0.56 \pm 0.06$ & $0.73 \pm 0.07$ & $1.0 \pm 0.1$ \\
\hline $\mathrm{Hf}$ & $0.029 \pm 0.009$ & $0.05 \pm 0.01$ & $0.04 \pm 0.01$ & $0.08 \pm 0.02$ & $0.11 \pm 0.02$ \\
\hline Cs & $0.140 \pm 0.007$ & $0.152 \pm 0.007$ & $0.086 \pm 0.004$ & $0.143 \pm 0.007$ & $0.154 \pm 0.007$ \\
\hline Th & $0.043 \pm 0.003$ & $0.067 \pm 0.004$ & $0.059 \pm 0.004$ & $0.109 \pm 0.007$ & $0.163 \pm 0.01$ \\
\hline $\mathrm{U}$ & $0.023 \pm 0.001$ & n.d. & $0.032 \pm 0.001$ & $0.047 \pm 0.002$ & $0.064 \pm 0.002$ \\
\hline $\mathrm{Cu}$ & $2.47 \pm 0.01$ & $3.15 \pm 0.01$ & $4.18 \pm 0.01$ & $4.53 \pm 0.01$ & $6.92 \pm 0.02$ \\
\hline $\mathrm{Pb}$ & $1.30 \pm 0.04$ & $1.08 \pm 0.03$ & $1.34 \pm 0.04$ & $1.28 \pm 0.04$ & $1.64 \pm 0.04$ \\
\hline $\mathrm{Cd}$ & $0.193 \pm 0.008$ & $0.153 \pm 0.008$ & $0.334 \pm 0.009$ & $0.169 \pm 0.008$ & $0.173 \pm 0.008$ \\
\hline Site & \multicolumn{5}{|c|}{ BG } \\
\hline Month & $\mathrm{I}$ & II & III & IV & $\mathrm{V}$ \\
\hline $\mathrm{Na}$ & $348 \pm 21$ & $341 \pm 20$ & $258 \pm 15$ & $673 \pm 40$ & $431 \pm 25$ \\
\hline $\mathrm{Mg}$ & $1030 \pm 61$ & $945 \pm 57$ & $1120 \pm 68$ & $924 \pm 55$ & $887 \pm 53$ \\
\hline $\mathrm{Cl}$ & $157 \pm 14$ & $413 \pm 37$ & $376 \pm 33$ & $353 \pm 31$ & $559 \pm 50$ \\
\hline $\mathrm{K}$ & $4400 \pm 350$ & $3730 \pm 300$ & $3280 \pm 260$ & $3470 \pm 280$ & $2750 \pm 220$ \\
\hline $\mathrm{Sc}$ & $0.048 \pm 0.002$ & $0.081 \pm 0.004$ & $0.059 \pm 0.003$ & $0.077 \pm 0.004$ & $0.077 \pm 0.004$ \\
\hline $\mathrm{Ca}$ & $5560 \pm 830$ & $4260 \pm 640$ & $5880 \pm 880$ & $4180 \pm 630$ & $4910 \pm 735$ \\
\hline $\mathrm{Ti}$ & $39 \pm 6$ & n.d. & n.d. & n.d. & n.d. \\
\hline $\mathrm{Cr}$ & $2.1 \pm 0.3$ & $1.0 \pm 0.2$ & $1.1 \pm 0.2$ & $1.0 \pm 0.2$ & $1.5 \pm 0.2$ \\
\hline $\mathrm{V}$ & $0.93 \pm 0.04$ & $0.78 \pm 0.03$ & $0.75 \pm 0.03$ & $0.77 \pm 0.03$ & $0.88 \pm 0.04$ \\
\hline $\mathrm{Mn}$ & $357 \pm 17$ & $303 \pm 15$ & $332 \pm 16$ & $232 \pm 12$ & $251 \pm 12$ \\
\hline $\mathrm{Ni}$ & $3.6 \pm 0.5$ & $1.3 \pm 0.2$ & $1.6 \pm 0.2$ & $1.2 \pm 0.2$ & $1.8 \pm 0.3$ \\
\hline $\mathrm{Fe}$ & $253 \pm 17$ & $338 \pm 23$ & $276 \pm 19$ & $329 \pm 23$ & $327 \pm 23$ \\
\hline $\mathrm{Co}$ & $0.19 \pm 0.01$ & $0.25 \pm 0.02$ & $0.27 \pm 0.02$ & $0.24 \pm 0.02$ & $0.26 \pm 0.02$ \\
\hline $\mathrm{Zn}$ & $44 \pm 1$ & $53 \pm 1$ & $49 \pm 1$ & $50 \pm 1$ & $55 \pm 1$ \\
\hline
\end{tabular}




\begin{tabular}{|c|c|c|c|c|c|}
\hline Site & \multicolumn{5}{|c|}{ BG } \\
\hline As & $1.20 \pm 0.08$ & $0.74 \pm 0.05$ & $0.61 \pm 0.04$ & $0.62 \pm 0.04$ & $0.65 \pm 0.04$ \\
\hline $\mathrm{Br}$ & $3.2 \pm 0.1$ & $4.4 \pm 0.1$ & $3.4 \pm 0.1$ & $3.9 \pm 0.1$ & $4.4 \pm 0.2$ \\
\hline $\mathrm{Sr}$ & $11 \pm 1$ & $22 \pm 1$ & $15 \pm 1$ & $17 \pm 1$ & $26 \pm 2$ \\
\hline $\mathrm{Rb}$ & $21 \pm 4$ & $16 \pm 3$ & $19 \pm 3$ & $12 \pm 2$ & $15 \pm 2$ \\
\hline Mo & $0.12 \pm 0.03$ & $0.13 \pm 0.04$ & $0.11 \pm 0.03$ & $0.09 \pm 0.03$ & $0.13 \pm 0.04$ \\
\hline $\mathrm{Sb}$ & $0.099 \pm 0.009$ & $0.30 \pm 0.02$ & $0.28 \pm 0.02$ & $0.28 \pm 0.02$ & $0.26 \pm 0.02$ \\
\hline $\mathrm{Ba}$ & $23 \pm 2$ & $23 \pm 2$ & $27 \pm 2$ & $15 \pm 1$ & $27 \pm 2$ \\
\hline $\mathrm{La}$ & $0.18 \pm 0.01$ & $0.27 \pm 0.01$ & $0.20 \pm 0.01$ & $0.34 \pm 0.01$ & $0.29 \pm 0.01$ \\
\hline $\mathrm{Ce}$ & $0.26 \pm 0.03$ & $0.56 \pm 0.05$ & $0.26 \pm 0.03$ & $0.45 \pm 0.05$ & $0.53 \pm 0.05$ \\
\hline $\mathrm{Hf}$ & $0.07 \pm 0.02$ & $0.05 \pm 0.01$ & $0.03 \pm 0.01$ & $0.03 \pm 0.01$ & $0.04 \pm 0.01$ \\
\hline $\mathrm{Cs}$ & $0.122 \pm 0.006$ & $0.096 \pm 0.006$ & $0.120 \pm 0.006$ & $0.108 \pm 0.006$ & $0.121 \pm 0.006$ \\
\hline Th & $0.037 \pm 0.002$ & $0.064 \pm 0.004$ & $0.043 \pm 0.002$ & $0.092 \pm 0.006$ & $0.061 \pm 0.004$ \\
\hline $\mathrm{U}$ & $0.014 \pm 0.001$ & $0.028 \pm 0.001$ & $0.015 \pm 0.001$ & $0.026 \pm 0.002$ & $0.027 \pm 0.002$ \\
\hline $\mathrm{Cu}$ & $3.55 \pm 0.01$ & $3.37 \pm 0.01$ & $3.85 \pm 0.01$ & $3.58 \pm 0.01$ & $3.58 \pm 0.01$ \\
\hline $\mathrm{Pb}$ & $0.97 \pm 0.03$ & $0.53 \pm 0.01$ & $1.36 \pm 0.03$ & $0.84 \pm 0.01$ & $1.55 \pm 0.03$ \\
\hline $\mathrm{Cd}$ & $0.214 \pm 0.008$ & $0.176 \pm 0.008$ & $0.167 \pm 0.008$ & $0.196 \pm 0.008$ & $0.257 \pm 0.009$ \\
\hline Site & \multicolumn{5}{|c|}{ AS } \\
\hline Month & I & II & III & IV & $\mathrm{V}$ \\
\hline $\mathrm{Na}$ & $393 \pm 25$ & $217 \pm 13$ & $786 \pm 45$ & $1530 \pm 90$ & $1050 \pm 63$ \\
\hline $\mathrm{Mg}$ & $725 \pm 43$ & $639 \pm 38$ & $980 \pm 59$ & $1170 \pm 70$ & $949 \pm 57$ \\
\hline $\mathrm{Cl}$ & $171 \pm 15$ & $264 \pm 24$ & $740 \pm 67$ & $1360 \pm 122$ & $987 \pm 88$ \\
\hline $\mathrm{K}$ & $3530 \pm 280$ & $816 \pm 65$ & $2100 \pm 170$ & $2330 \pm 180$ & $3880 \pm 310$ \\
\hline $\mathrm{Sc}$ & $0.08 \pm 0.004$ & $0.15 \pm 0.008$ & $0.26 \pm 0.01$ & $0.18 \pm 0.09$ & $0.20 \pm 0.01$ \\
\hline $\mathrm{Ca}$ & $10700 \pm 1600$ & $11000 \pm 1650$ & $7970 \pm 1120$ & $11000 \pm 1650$ & $6310 \pm 940$ \\
\hline $\mathrm{Ti}$ & $82 \pm 12$ & $32 \pm 5$ & $96 \pm 14$ & $69 \pm 10$ & $36 \pm 5$ \\
\hline $\mathrm{Cr}$ & $2.4 \pm 0.4$ & $1.9 \pm 0.3$ & $4.0 \pm 0.6$ & $2.9 \pm 0.4$ & $3.2 \pm 0.5$ \\
\hline $\mathrm{V}$ & $0.96 \pm 0.05$ & $0.79 \pm 0.04$ & $1.94 \pm 0.06$ & $1.22 \pm 0.06$ & $2.20 \pm 0.07$ \\
\hline $\mathrm{Mn}$ & $230 \pm 11$ & $192 \pm 10$ & $264 \pm 13$ & $359 \pm 18$ & $334 \pm 17$ \\
\hline $\mathrm{Ni}$ & $0.7 \pm 0.1$ & $0.7 \pm 0.1$ & $2.2 \pm 0.3$ & $1.5 \pm 0.2$ & $3.1 \pm 0.5$ \\
\hline $\mathrm{Fe}$ & $324 \pm 23$ & $446 \pm 31$ & $942 \pm 65$ & $707 \pm 50$ & $778 \pm 55$ \\
\hline $\mathrm{Co}$ & $0.33 \pm 0.03$ & $0.30 \pm 0.03$ & $0.57 \pm 0.05$ & $0.54 \pm 0.05$ & $0.53 \pm 0.05$ \\
\hline $\mathrm{Zn}$ & $47 \pm 1$ & $46 \pm 1$ & $67 \pm 2$ & $61 \pm 2$ & $64 \pm 2$ \\
\hline As & $0.21 \pm 0.01$ & $0.23 \pm 0.01$ & $0.37 \pm 0.03$ & $0.38 \pm 0.03$ & $0.42 \pm 0.03$ \\
\hline $\mathrm{Br}$ & $6.5 \pm 0.2$ & $5.0 \pm 0.1$ & $7.9 \pm 0.2$ & $9.5 \pm 0.2$ & $6.9 \pm 0.1$ \\
\hline $\mathrm{Sr}$ & $24 \pm 2$ & $52 \pm 5$ & $45 \pm 4$ & $35 \pm 3$ & $41 \pm 4$ \\
\hline $\mathrm{Rb}$ & $16 \pm 3$ & $3 \pm 1$ & $10 \pm 2$ & $12 \pm 2$ & $11 \pm 2$ \\
\hline Mo & $0.10 \pm 0.03$ & $0.13 \pm 0.04$ & $0.30 \pm 0.09$ & $0.26 \pm 0.08$ & $0.21 \pm 0.06$ \\
\hline $\mathrm{Sb}$ & $0.47 \pm 0.04$ & $0.91 \pm 0.08$ & $1.3 \pm 0.1$ & $0.95 \pm 0.08$ & $0.61 \pm 0.05$ \\
\hline $\mathrm{Ba}$ & $19 \pm 2$ & $16 \pm 1$ & $27 \pm 3$ & $27 \pm 3$ & $31 \pm 3$ \\
\hline $\mathrm{La}$ & $0.28 \pm 0.01$ & $0.32 \pm 0.01$ & $0.64 \pm 0.03$ & $0.64 \pm 0.03$ & $0.66 \pm 0.03$ \\
\hline $\mathrm{Ce}$ & $0.26 \pm 0.03$ & $0.50 \pm 0.05$ & $1.3 \pm 0.1$ & $1.5 \pm 0.1$ & $1.0 \pm 0.1$ \\
\hline $\mathrm{Hf}$ & $0.04 \pm 0.01$ & $0.09 \pm 0.02$ & $0.14 \pm 0.04$ & $0.09 \pm 0.02$ & $0.08 \pm 0.02$ \\
\hline Cs & $0.095 \pm 0.002$ & $0.072 \pm 0.001$ & $0.120 \pm 0.005$ & $0.107 \pm 0.005$ & $0.108 \pm 0.005$ \\
\hline Th & $0.066 \pm 0.004$ & $0.081 \pm 0.005$ & $0.17 \pm 0.01$ & $0.17 \pm 0.01$ & $0.17 \pm 0.01$ \\
\hline $\mathrm{U}$ & $0.047 \pm 0.004$ & $0.15 \pm 0.01$ & $0.14 \pm 0.01$ & $0.077 \pm 0.006$ & $0.079 \pm 0.006$ \\
\hline $\mathrm{Cu}$ & $5.36 \pm 0.02$ & $4.93 \pm 0.01$ & $8.59 \pm 0.03$ & $8.30 \pm 0.03$ & $5.33 \pm 0.02$ \\
\hline $\mathrm{Pb}$ & $0.71 \pm 0.02$ & $1.90 \pm 0.06$ & $1.69 \pm 0.06$ & $1.42 \pm 0.04$ & $1.41 \pm 0.04$ \\
\hline $\mathrm{Cd}$ & $0.148 \pm 0.007$ & $0.157 \pm 0.008$ & $0.139 \pm 0.007$ & $0.187 \pm 0.009$ & $0.195 \pm 0.009$ \\
\hline
\end{tabular}

Data presented in Vukovic et al. [16] study showed that RAF values higher than 0.5 indicated slight elemental enrichment in the moss, and the values higher than one indicated considerable elemental enrichment. 
The RAFs for $\mathrm{Na}, \mathrm{Sc}, \mathrm{Ca}, \mathrm{V}, \mathrm{Cr}, \mathrm{Ni}, \mathrm{Fe}, \mathrm{Co}, \mathrm{Sr}, \mathrm{Sb}, \mathrm{Ba}, \mathrm{La}, \mathrm{Ce}, \mathrm{Hf}, \mathrm{Th}, \mathrm{Zn}$, and Cd at TPP site had the values higher than one. RAFs for $\mathrm{V}$, La and Sb reached the highest values of 6.2, 11.3 and 13.5, respectively. Vanadium and $\mathrm{Ni}$ are the two trace metals emitted in large amounts during the oil combustion $[11,17]$. Elements such as $\mathrm{Sb}, \mathrm{As}, \mathrm{U}$, and $\mathrm{Cr}$ are considered as indicators of emission from fossil fuel combustion processes. The moss samples were exposed in heating seasons to verify whether the high accumulation of the abovementioned elements, being components of crude oil or coal, could occur. The TPP zone is also surrounded by heavy traffic roads. The elements found in the moss samples from this site, such as $\mathrm{Ca}, \mathrm{Fe}, \mathrm{Co}, \mathrm{Zn}, \mathrm{Mo}, \mathrm{Sb}$, and $\mathrm{Ba}$, are characteristic for road traffic [11, 18]. Some of elements in moss, namely $\mathrm{La}, \mathrm{Ce}, \mathrm{Hf}$, and Th may originate from the re-suspension of soil and road dust [4].

Table 2

Min, max, mean, median $\left[\mathrm{mg} \mathrm{kg}^{-1}\right]$ and RAF values of 30 elements determined by NAA (27 elements) and $\mathrm{AAS}(\mathrm{Cu}, \mathrm{Cd}$ and $\mathrm{Pb})$

\begin{tabular}{|c|c|c|c|c|c|c|c|c|}
\hline Site & \multicolumn{3}{|c|}{ TPP } & \multicolumn{3}{|c|}{ BG } \\
\hline Element & Min & Max & Median & $\mathbf{R A F}_{\text {median }}$ & Min & Max & Median & RAF $_{\text {median }}$ \\
\hline $\mathbf{N a}$ & 269 & 2720 & 608 & 3.5 & 258 & 673 & 348 & 1.6 \\
\hline $\mathbf{M g}$ & .d. & 1040 & 0 & - & 887 & 1120 & 945 & 10.3 \\
\hline $\mathbf{C l}$ & 394 & 2430 & 568 & 0 & 157 & 559 & 376 & 0 \\
\hline $\mathbf{K}$ & 2440 & 6890 & 5020 & 0 & 2750 & 4400 & 3470 & 0 \\
\hline $\mathbf{S c}$ & 0.057 & 0.174 & 0.09 & 2.7 & 0.0 & 0.08 & 0.08 & 2.2 \\
\hline $\mathbf{C a}$ & 2980 & 9680 & 6500 & 1.4 & 4180 & 5880 & 4910 & 0.8 \\
\hline $\mathbf{T i}$ & 31 & 110 & 72 & 0 & 0 & 38.7 & 0 & 0 \\
\hline $\mathbf{V}$ & 2.0 & 10.8 & 3.9 & 6.2 & 0.8 & 0.9 & 0.8 & 0.5 \\
\hline $\mathbf{C r}$ & 0.7 & 2.3 & 2.0 & 1.6 & 1 & 2.05 & 303 & 0.4 \\
\hline $\mathbf{M n}$ & 239 & 390 & 295 & 0.2 & 232 & 357 & 1.6 & 0.2 \\
\hline $\mathbf{N i}$ & 1.5 & 4.7 & 2.7 & 2.4 & 1.2 & 3.57 & 327 & 1.0 \\
\hline $\mathbf{F e}$ & 276 & 646 & 378 & 1.8 & 253 & 338 & 0.3 & 1.4 \\
\hline $\mathbf{C o}$ & 0.3 & 0.5 & 0.3 & 1.3 & 0.19 & 0.27 & 49.6 & 0.7 \\
\hline $\mathbf{Z n}$ & 43 & 88 & 54 & 1.0 & 44.3 & 54.5 & 0.7 & 0.8 \\
\hline $\mathbf{A s}$ & 0.7 & 1.2 & 1.0 & 0.5 & 0.61 & 1.2 & 3.9 & 0 \\
\hline $\mathbf{B r}$ & 3.5 & 7.1 & 5.9 & 0.7 & 3.17 & 4.38 & 16.8 & 0.1 \\
\hline $\mathbf{S r}$ & 10 & 35 & 24 & 2.9 & 11.2 & 25.5 & 16.1 & 1.8 \\
\hline $\mathbf{R b}$ & 11 & 31 & 26 & 0 & 12.3 & 21.3 & 0.1 & 0 \\
\hline $\mathbf{M o}$ & 0.11 & 0.22 & 0.17 & 0 & 0.09 & 0.13 & 0.3 & 0 \\
\hline $\mathbf{S b}$ & 0.25 & 1 & 0.4 & 13.5 & 0.10 & 0.30 & 23.3 & 8.4 \\
\hline $\mathbf{B a}$ & 18 & 29 & 28 & 1.8 & 14.9 & 26.9 & 0.3 & 1.3 \\
\hline $\mathbf{L a}$ & 0.2 & 0.6 & 0.3 & 11.3 & 0.18 & 0.34 & 0.5 & 10.6 \\
\hline $\mathbf{C e}$ & 0.3 & 1.0 & 0.7 & 1.4 & 0.26 & 0.56 & 0.0 & 0.5 \\
\hline $\mathbf{H f}$ & 0.03 & 0.11 & 0.05 & 2.7 & 0.03 & 0.07 & 0.1 & 1.7 \\
\hline $\mathbf{T h}$ & 0.04 & 0.16 & 0.07 & 2.9 & 0.04 & 0.09 & 0.06 & 2.5 \\
\hline $\mathbf{U}$ & n.d. & 0.06 & 0.03 & 3.1 & 0.01 & 0.03 & 3.6 & 2.3 \\
\hline $\mathbf{C u}$ & 2.5 & 6.9 & 4.2 & 0.3 & 3.37 & 3.85 & 1.0 & 0.1 \\
\hline $\mathbf{P b}$ & 1.1 & 1.6 & 1.3 & 1.6 & 0.53 & 1.55 & 0.2 & 0.9 \\
\hline $\mathbf{C d}$ & 0.2 & 0.3 & 0.2 & 0.6 & 0.17 & 0.26 & 1.1 & 0.9 \\
\hline & & & & & & & & \\
\hline
\end{tabular}

\begin{tabular}{|c|c|c|c|c|}
\hline Site & \multicolumn{3}{|c|}{ AS } \\
\hline Element & Min & Max & Median & RAF $_{\text {median }}$ \\
\hline Na & 217 & 1530 & 786 & 4.9 \\
\hline Mg & 639 & 1170 & 949 & 10.3 \\
\hline Cl & 171 & 1360 & 740 & 0.3 \\
\hline K & 816 & 3880 & 2330 & 0 \\
\hline
\end{tabular}




\begin{tabular}{|c|c|c|c|c|}
\hline Site & \multicolumn{4}{|c|}{ AS } \\
\hline Element & Min & Max & Median & RAF $_{\text {median }}$ \\
\hline $\mathbf{S c}$ & 0.1 & 0.3 & 0.2 & 6.3 \\
\hline $\mathbf{C a}$ & 6310 & 11000 & 10700 & 3.0 \\
\hline $\mathbf{T i}$ & 32 & 96 & 69 & 0 \\
\hline $\mathbf{V}$ & 0.8 & 2.2 & 1.3 & 1.4 \\
\hline $\mathbf{C r}$ & 1.9 & 4.0 & 2.9 & 3.0 \\
\hline $\mathbf{M n}$ & 192 & 359 & 264 & 0.1 \\
\hline $\mathbf{N i}$ & 0.7 & 3.1 & 1.5 & 0.8 \\
\hline $\mathbf{F e}$ & 324 & 942 & 707 & 4.3 \\
\hline $\mathbf{C o}$ & 0.30 & 0.57 & 0.53 & 2.7 \\
\hline $\mathbf{Z n}$ & 46 & 67 & 61 & 1.2 \\
\hline $\mathbf{A s}$ & 0.2 & 0.4 & 0.37 & 0 \\
\hline $\mathbf{B r}$ & 5.0 & 9.5 & 6.9 & 1.0 \\
\hline $\mathbf{S r}$ & 24 & 52 & 41 & 5.8 \\
\hline $\mathbf{R b}$ & 3.2 & 16 & 10.6 & 0 \\
\hline $\mathbf{M o}$ & 0.1 & 0.3 & 0.2 & 0.1 \\
\hline $\mathbf{S b}$ & 0.5 & 1.3 & 0.9 & 29 \\
\hline $\mathbf{B a}$ & 16 & 31 & 27 & 1.7 \\
\hline $\mathbf{L a}$ & 0.3 & 0.7 & 0.6 & 29 \\
\hline $\mathbf{C e}$ & 0.3 & 1.5 & 1.0 & 2.4 \\
\hline $\mathbf{H f}$ & 0.04 & 0.14 & 0.09 & 5.2 \\
\hline $\mathbf{T h}$ & 0.07 & 0.17 & 0.17 & 8.6 \\
\hline $\mathbf{U}$ & 0.05 & 0.15 & 0.08 & 9.2 \\
\hline $\mathbf{C u}$ & 4.9 & 8.6 & 5.4 & 0.7 \\
\hline $\mathbf{P b}$ & 0.7 & 1.9 & 1.4 & 1.8 \\
\hline $\mathbf{C d}$ & 0.14 & 0.19 & 0.16 & 0.5 \\
\hline & & & & \\
\hline
\end{tabular}

Table 3

Trace element concentrations [ $\mathrm{mg} \mathrm{kg}^{-1} \mathrm{~d} . \mathrm{m}$.] in studied unexposed moss samples in comparison with literature data

\begin{tabular}{|c|c|c|c|c|}
\hline Element & \multicolumn{5}{|c|}{ Moss collection place and year } \\
\hline & $\begin{array}{c}\text { Tver region, } \\
\text { Russia, 2016 }\end{array}$ & $\begin{array}{c}\text { Vitosha Mountain Natural } \\
\text { Park, Bulgaria, 2001 [6] }\end{array}$ & $\begin{array}{c}\text { Tver region, } \\
\text { Russia, 2013 [17] }\end{array}$ & $\begin{array}{c}\text { Tver region, Russia, } \\
\text { 2005 [4] }\end{array}$ \\
\hline $\mathbf{N a}$ & $134 \pm 8$ & 241 & 58 & 197 \\
\hline $\mathbf{M g}$ & $84 \pm 5$ & 1226 & 1436 & - \\
\hline $\mathbf{C l}$ & $579 \pm 50$ & 1025 & 9912 & 11500 \\
\hline $\mathbf{K}$ & $10800 \pm 860$ & 6699 & - & 0.033 \\
\hline $\mathbf{S c}$ & $0.024 \pm 0.001$ & 0.22 & 3298 & 2300 \\
\hline $\mathbf{C a}$ & $2680 \pm 400$ & 2663 & - & - \\
\hline $\mathbf{T i}$ & $78 \pm 11$ & 66 & 0.4 & 0.26 \\
\hline $\mathbf{C r}$ & $0.7 \pm 0.1$ & 1.7 & 221 & 0.5 \\
\hline $\mathbf{V}$ & $0.54 \pm 0.06$ & 2.1 & 1.7 & 2.5 \\
\hline $\mathbf{M n}$ & $243 \pm 12$ & 173 & 300 & 297 \\
\hline $\mathbf{N i}$ & $0.8 \pm 0.1$ & 1.4 & 0.36 & 0.37 \\
\hline $\mathbf{F e}$ & $134 \pm 9$ & 864 & 24.1 & 21 \\
\hline $\mathbf{C o}$ & $0.14 \pm 0.01$ & 0.33 & - & 0.11 \\
\hline $\mathbf{Z n}$ & $28 \pm 1$ & 36 & - & 3.4 \\
\hline $\mathbf{A s}$ & $0.71 \pm 0.05$ & 0.67 & 6.5 & 7.6 \\
\hline $\mathbf{B r}$ & $3.4 \pm 0.1$ & 4.2 & - & 71 \\
\hline $\mathbf{S r}$ & $6 \pm 1$ & 26 & - & - \\
\hline $\mathbf{R b}$ & $58 \pm 10$ & 15 & - & 0.04 \\
\hline $\mathbf{M o}$ & $0.202 \pm 0.006$ & - & 27.7 & 17 \\
\hline $\mathbf{S b}$ & $0.029 \pm 0.003$ & 0.095 & 56 & \\
\hline $\mathbf{B a}$ & $10 \pm 1$ & & & \\
\hline & & 56 & & \\
\hline
\end{tabular}




\begin{tabular}{|c|c|c|c|c|}
\hline Element & \multicolumn{4}{|c|}{ Moss collection place and year } \\
\hline & $\begin{array}{c}\text { Tver region, } \\
\text { Russia, 2016 }\end{array}$ & $\begin{array}{c}\text { Vitosha Mountain Natural } \\
\text { Park, Bulgaria, 2001 [6] }\end{array}$ & $\begin{array}{c}\text { Tver region, } \\
\text { Russia, 2013 [17] }\end{array}$ & $\begin{array}{c}\text { Tver region, Russia, } \\
\mathbf{2 0 0 5}[\mathbf{4}]\end{array}$ \\
\hline La & $0.023 \pm 0.001$ & 0.6 & - & 0.19 \\
\hline Ce & $0.30 \pm 0.03$ & - & - & 0.38 \\
\hline Hf & $0.014 \pm 0.004$ & 0.078 & - & - \\
\hline Th & $0.018 \pm 0.001$ & 0.19 & - & 0.027 \\
\hline $\mathbf{U}$ & $0.008 \pm 0.001$ & 0.094 & 2.6 & 0.015 \\
\hline $\mathbf{C u}$ & $3.2 \pm 0.1$ & 7.6 & 2.14 & - \\
\hline Pb & $0.51 \pm 0.02$ & - & 0.17 & - \\
\hline Cd & $0.106 \pm 0.005$ & 0.3 & & - \\
\hline
\end{tabular}

At the BG, the RAF values higher than one were obtained for $\mathrm{Na}, \mathrm{Mg}, \mathrm{Sc}, \mathrm{Ni}, \mathrm{Fe}, \mathrm{Sr}$, $\mathrm{Sb}, \mathrm{Ba}, \mathrm{La}, \mathrm{Hf}, \mathrm{Th}$, and U. Some of the elements ( $\mathrm{Na}, \mathrm{Mg}, \mathrm{Ba}, \mathrm{Hf}$, and $\mathrm{Sr}$ ) may also originate from the re-suspension of soil and road dust [19]. Magnesium may occur due to leaching of living or dead tissue of other plants [20]. The main sources of the elements such as $\mathrm{Ni}, \mathrm{Fe}, \mathrm{Sb}$, and $\mathrm{Cd}$ can be associated to the transport and industrial activity [21, 22]. The Botanical garden is situated in the vicinity of the city and it is surrounded by the national roads.

At the AS site, being a central city zone, the RAF values for $\mathrm{Na}, \mathrm{Mg}, \mathrm{Sc}, \mathrm{Ca}, \mathrm{V}, \mathrm{Cr}$, $\mathrm{Co}, \mathrm{Fe}, \mathrm{Zn}, \mathrm{Br}, \mathrm{Sb}, \mathrm{Sr}, \mathrm{Ba}, \mathrm{La}, \mathrm{Ce}, \mathrm{Hf}, \mathrm{W}, \mathrm{Th}, \mathrm{U}$, and $\mathrm{Pb}$ were higher than one. The increase of $\mathrm{Fe}, \mathrm{Cr}, \mathrm{Sc}$, Th, and $\mathrm{U}$ content is an indication of the input of terrigenous dust to the moss [23]. Some elements found in moss from this site, such as $\mathrm{Ca}, \mathrm{Cr}, \mathrm{Fe}, \mathrm{Co}, \mathrm{Zn}, \mathrm{Br}, \mathrm{Sb}$, and $\mathrm{Ba}$, are characteristic for the road traffic [11]. The RAF values for $\mathrm{Pb}$ were higher than one at TTP and AS sites. The main source of $\mathrm{Pb}$ emissions is the use of leaded gasoline combustion [18]. Negative RAF values were obtained for the physiologically active elements such as $\mathrm{Cl}$ (TPP and BG), $\mathrm{K}$ and the alkali elements as Rb (at all sites). The behaviour of $\mathrm{Rb}$ in plants is similar to that of $\mathrm{K}$ and $\mathrm{Cl}$ indicates the damage of the moss cell membrane due to the impact of air pollutants [4]. The loss of $\mathrm{K}$ from the exposed moss tissue was also noticed in the other studies employing moss bags $[4,16]$. Negative RAF values may also result from the uncertainty of the measurement method. In Table 2, negative RAF values were assumed to be RAF $=0$.

For the elements considered as environmental contaminants, the concentration in the exposed moss samples was significantly higher than in the background ones (the initial moss element concentrations), indicating that the sites at which the moss bags were exposed were polluted by the determined elements (Fig. 2).

The behaviour of these elements differs at different sites of exposure. The most pronounced increase of $\mathrm{V}$ content was observed at the TPP. As it has been previously mentioned, $\mathrm{V}$ and $\mathrm{Ni}$ are two trace elements emitted in large amounts during the oil combustion. Vanadium is also present in coal. The moss accumulated the maximal amount of $\mathrm{V}$ after 4 months of exposure and then its content decreased threefold. The high content of $\mathrm{V}$ in January and February can be explained by low outside temperatures and consequently, more intense heating. Vanadium content almost did not change at the BG site. At AS site its bioaccumulation can be explained by windblown dust as well as in vicinity of the TPP site. The increase of Ni content at all exposure sites was observed. Major anthropogenic sources of Ni release are the combustion of coal and heavy fuel oil $[18,24]$. It was shown in Anicic et al. [4] study that $\mathrm{V}$ and $\mathrm{Ni}$ content in the winter time (corresponding to the official heating season) was two and three times higher than in the summer time, and for As and $\mathrm{Fe}$ it was 1.5 times higher in winter. A continuous increase of 
As content at the TPP site was detected. It is well known, that the main As pollution sources are the high-temperature oil and coal burning, as well as the operation of power plants [25]. At the BG site (except the first month) and the AS site, As content was lower than in the control sample.

a)

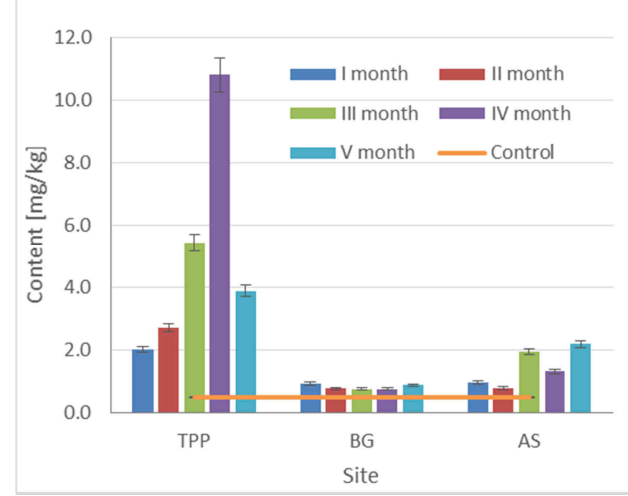

c)

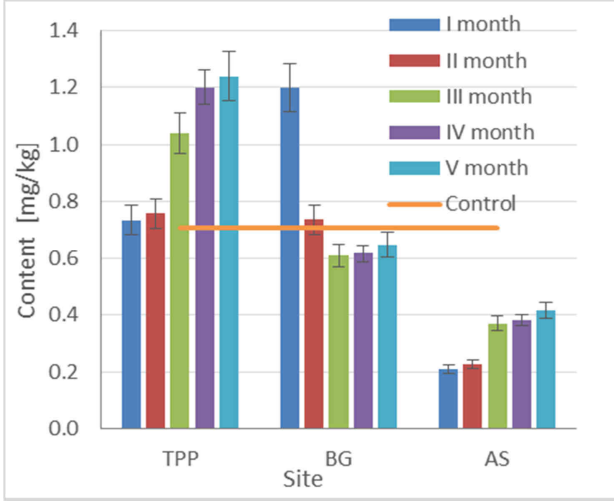

e)

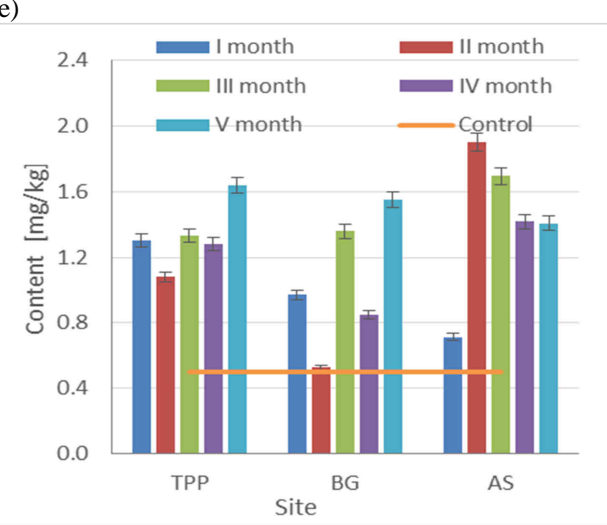

b)

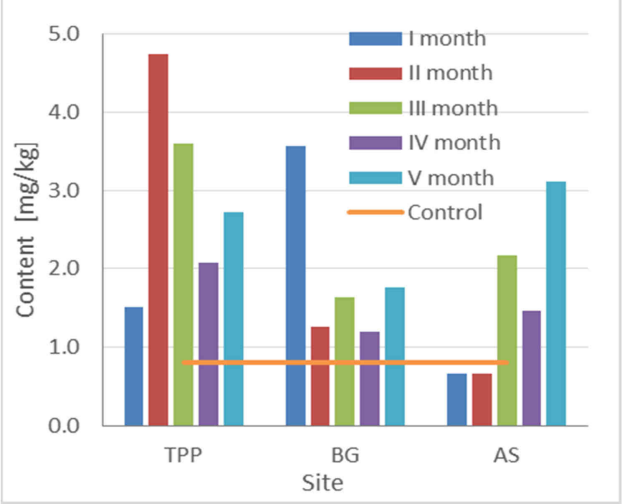

d)

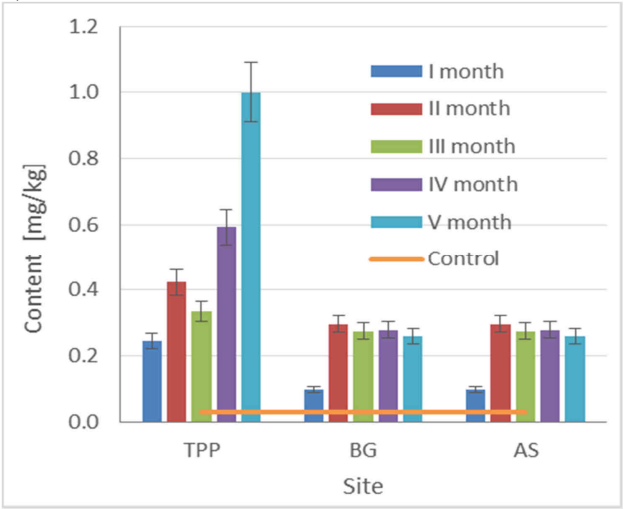

f)

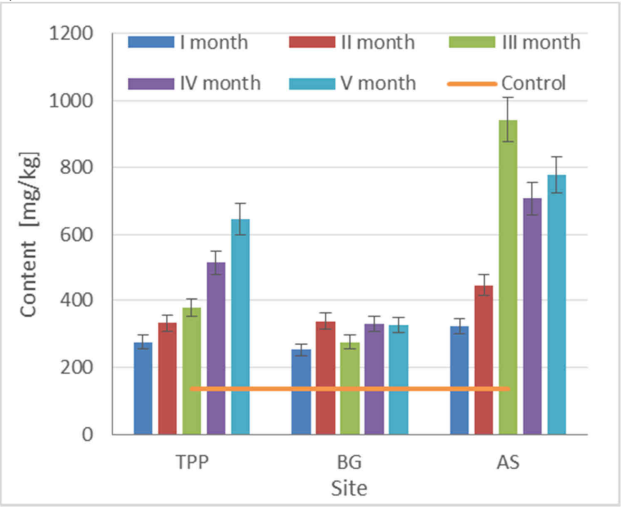

Fig. 2. Concentrations of the elements in moss bags exposed for 5 months at three sites in Chisinau: a) vanadium, b) nickel, c) arsenic, d) antimony, e) lead, f) iron 
The high content of $\mathrm{Sb}$ at TPP site can be explained by coal burning and traffic emissions, a key tracer of non-exhaust traffic, i.e., deterioration of tires, brakes, engines and vehicle components [26]. High concentrations of $\mathrm{Sb}$ at the BG and AS sites could be related with its naturally release from the Earth's crust. The increase of $\mathrm{Pb}$ content at all three sites indicates the use of lead-loaded gasoline in the Republic of Moldova. Emissions from traffic, besides $\mathrm{Pb}$, contain many potentially toxic elements such as, $\mathrm{Cd}$, and $\mathrm{Zn}$. Iron is generally considered as a typical geological marker element. However, in urban areas, it often originates from metallic wear parts of vehicles and road dust re-suspension [11]. In case of some elements decrease of their concentration after several months of accumulation was observed. This decrease can be explained in several ways: (i) precipitation (snow) as well as wind blow could remove easily captured particles or (ii) frost could lead to membrane disruption and leakage of already accumulated elements. However, as decrease of metal content was observed not at all sites wind and precipitations can be considered as main factors affecting metal accumulation by moss samples.

The results of principal component analysis are presented in Table 4. Three factors explaining a total of $81 \%$ of the variability were identified in the data set. The first factor, which elucidates most of the variance (49\%), has high loadings for $\mathrm{Cr}(0.87)$, Fe (0.94), $\mathrm{Co}(0.92), \mathrm{Sr}(0.92), \mathrm{Sb}(0.96), \mathrm{U}(0.89)$, and $\mathrm{Cu}(0.90)$. This factor represents a combined geogenic and anthropogenic association of the elements.

Table 4

Factor loadings after Varimax rotation for the elements determined in exposed moss samples

\begin{tabular}{|c|c|c|c|}
\hline & F1 & F2 & F3 \\
\hline $\mathbf{A l}$ & 0.43 & $\mathbf{0 . 8 7}$ & 0.05 \\
\hline $\mathbf{C r}$ & $\mathbf{0 . 8 7}$ & 0.10 & 0.05 \\
\hline $\mathbf{V}$ & 0.15 & $\mathbf{0 . 8 6}$ & 0.13 \\
\hline $\mathbf{N i}$ & -0.04 & $\mathbf{0 . 4 3}$ & 0.56 \\
\hline $\mathbf{F e}$ & $\mathbf{0 . 9 4}$ & 0.13 & 0.10 \\
\hline $\mathbf{C o}$ & $\mathbf{0 . 9 2}$ & 0.19 & 0.11 \\
\hline $\mathbf{Z n}$ & 0.48 & 0.17 & $\mathbf{0 . 7 6}$ \\
\hline $\mathbf{A s}$ & -0.27 & $\mathbf{0 . 8 3}$ & 0.19 \\
\hline $\mathbf{S r}$ & $\mathbf{0 . 9 2}$ & -0.06 & 0.06 \\
\hline $\mathbf{S b}$ & $\mathbf{0 . 9 6}$ & 0.08 & -0.10 \\
\hline $\mathbf{U}$ & $\mathbf{0 . 8 9}$ & -0.17 & -0.12 \\
\hline $\mathbf{C u}$ & $\mathbf{0 . 9 0}$ & 0.10 & -0.08 \\
\hline Pb & $\mathbf{0 . 7 0}$ & 0.08 & 0.27 \\
\hline $\mathbf{C d}$ & -0.14 & 0.05 & $\mathbf{0 . 9 3}$ \\
\hline Tot. Var. & 0.49 & 0.18 & 0.14 \\
\hline
\end{tabular}

Iron, $\mathrm{Sr}, \mathrm{Sb}$, and $\mathrm{U}$ are typical crustal elements, and their content in the moss can be associated with mineral particles released into the atmosphere mainly by wind erosion. However, high values for $\mathrm{Cr}, \mathrm{Fe}, \mathrm{Cu}$, and $\mathrm{Pb}$ also indicate the influence of anthropogenic sources. The second factor, with $18 \%$ of the total variance, shows high loading for $\mathrm{Al}, \mathrm{V}$, and As pointing to the contribution from thermal power plants. The third accounts for $14 \%$, with pronounced contribution from $\mathrm{Cd}(0.93)$ and $\mathrm{Zn}(0.76)$, might be attributed to traffic sources [18]. 


\section{Conclusions}

The concentrations of 30 elements were determined in the moss samples exposed at three sites in Chisinau city using NAA and AAS. Regarding the element content of the unexposed moss, a significant accumulation of the majority of examined elements in the S. girgensohnii moss bags was observed over the 5-month exposure periods indicating that this moss species is an efficient trace element accumulator in urban area. The RAF values and factor analysis support hypothesis that the primary sources of air pollution in Chisinau are vehicles, thermal power plants and industry. Obtained results showed high accumulation of potentially toxic elements by the moss samples, which indicates that serious air pollution in Chisinau exists and requires attention from the national authorities.

\section{References}

[1] Culicov OA, Duliu OG, Zinicovscaia I. Study of elemental grouping in moss-bags as a function of height and location of the exposure site. Rom Rep Phys. 2016;68(2):736-745. http://www.rrp.infim.ro/2016_68_2/A28.pdf.

[2] Modzelewska D, Dołhańczuk-Śródka A, Ziembik Z. Assessment of air contamination with selected radioisotopes nearby cement plant using moss bag method. Ecol Chem Eng S. 2015;22:447-455. DOI: 10.2428/ecea.2015.22(4)35.

[3] Gatziolis D, Jovan S, Donovan G, Amacher M, Monleon V. Elemental atmospheric pollution assessment via moss-based measurements in Portland, Oregon. Gen. Tech. Rep. PNW-GTR-938. Portland, OR: U.S. Department of Agriculture, Forest Service, Pacific Northwest Research Station. 2016. www.fs.usda.gov/treesearch/pubs/51076.

[4] Aničić M, Tasić M, Frontasyeva MV, Tomašević M, Rajšić S, Strelkova LP, et al. Active biomonitoring with wet and dry moss: a case study in an urban area. Environ Chem Lett. 2009;7(1):55-60. DOI: 10.1007/s10311-008-0135-4.

[5] Giordano S, Adamo P, Sorbo S, Vingiani S. Atmospheric trace metal pollution in the Naples urban area based on results from moss and lichen bags. Environ Pollut. 2005;136:431-442. DOI: 10.1016/j.envpol.2005.01.017.

[6] Culicov OA, Yurukova L. Comparison of element accumulation of different moss-and lichen-bags, exposed in the city of Sofia (Bulgaria). J Atmos Chem. 2006;55:1-12. DOI: 10.1007/s10874-005-9002-x.

[7] Fernández JA, Ares A, Rey-Asensio A, Carballeira A, Aboal JR. Effect of growth on active biomonitoring with terrestrial mosses. J Atmos Chem. 2009;63(1):1-11. DOI: 10.1007/s10874-010-9152-3.

[8] Anonymous. Republic of Moldova State of the Environment: Report 2006/ Ministry of Ecology and Natural Resources. Chisinau: Moldovan Academy of Sciences Institute of Ecology and Geography. 2007. www.unenvironment.org/resources/report/state-environment-report-moldova.

[9] Cojocaru A. Air pollution in the Republic of Moldova: current status and future prospects. In: Barnes I, Kharytonov MM, editors. Simulation and Assessment of Chemical Processes in a Multiphase Environment. NATO Science for Peace and Security Series C: Environmental Security. Dordrecht: Springer; 2008.

[10] Begu A. AGROFOR Int J. 2017;2:65-77. http://www.agrofor.rs.ba/data/20171115-08-Begu $\% 20$ at\%20all.pdf.

[11] Aničić M, Frontasyeva MV, Tomašević M, Popović A. Assessment of atmospheric deposition of heavy metals and other elements in Belgrade using the moss biomonitoring technique and neutron activation analysis. Environ Monit Assess. 2007;129:207-219. DOI: 10.1007/s10661-006-9354-y.

[12] Zinicovscaia I, Hramco C, Duliu OG, Vergel K, Culicov OA, Frontasyeva MV, et al. Air pollution study in the Republic of Moldova using moss biomonitoring technique. Bull Environ Contam Toxicol. 2017;98(2):262-269. DOI: 10.1007/s00128-016-1989-y.

[13] Frontasyeva MV. Neutron activation analysis in the life sciences. PEPAN. 2011;42(2):332-378. DOI: 10.1134/S1063779611020043.

[14] Pavlov SS, Dmitriev AY, Frontasyeva MV. Automation system for neutron activation analysis at the reactor IBR-2, Frank Laboratory of Neutron Physics, Joint Institute for Nuclear Research, Dubna, Russia. J Radioanal Nucl Chem. 2016;309:27-38. DOI: 10.1007/s10967-016-4864-8.

[15] Culicov OA, Mocanu R, Frontasyeva MV, Yurukova L, Steinnes E. Active moss biomonitoring applied to an industrial site in Romania: relative accumulation of 36 elements in moss-bags. Environ Monit Assess. 2005;108:229-240. DOI: 10.1007/s10661-005-1688-9. 
[16] Vuković G, Aničić Urošević M, Škrivanj S, Vergel K, Tomašević M, Popović A. The first survey of airborne trace elements at airport using moss bag technique. Environ Sci Pollut Res Int. 2017;24(17):15107-15115. DOI: $10.1007 / \mathrm{s} 11356-017-9140-0$.

[17] Pacyna JM, Pacyna EG. An assessment of global and regional emissions of trace metals to the atmosphere from anthropogenic sources worldwide. Environ Rev. 2001;9:269-298. DOI: 10.1139/a01-012.

[18] Frontasyeva MV, Steinnes E, Harmens H. Monitoring long-term and large-scale deposition of air pollutants based on moss analysis. In: Aničić M, Urošević M, Vuković G, Tomašević M, editors. Biomonitoring of Air Pollution Using Mosses and Lichens: Passive and Active Approach - State of the Art and Perspectives. NewYork: Nova Science Publishers; 2017.

[19] Shirmohammadi F, Hasheminassab S, Wang D, Saffari A, Schauer JJ, Shafer MM, et al. Oxidative potential of coarse particulate matter $\left(\mathrm{PM}_{10-2.5}\right)$ and its relation to water solubility and sources of trace elements and metals in the Los Angeles Basin. Environ Sci Process Impact. 2015;17(12):2110-2121. DOI: 10.1039/c5em00364d.

[20] Markert B. Establishing of 'Reference Plant' for inorganic characterization of different plant species by chemical fingerprinting. Water Air Soil Pollut. 1992;64(3):533-538. DOI: 10.1007/BF00483363.

[21] Siudek P, Frankowski M, Siepak J. Trace element distribution in the snow cover from an urban area in central Poland. Environ Monit Assess. 2015;187(5):225. DOI: 10.1007/s10661-015-4446-1.

[22] Kłos A, Ziembik Z, Rajfur M, Dołhańczuk-Śródka A, Bochenek Z, Bjerke JW, et al. The origin of heavy metals and radionuclides accumulated in the soil and biota samples collected in Svalbard, near Longyearbyen. Ecol Chem Eng S. 2017;24(2):223-238. DOI: 10.1515/eces-2017-0015.

[23] Reimann C, Niskavaara H, Kashulina G, Filzmoser P, Boyd R, Volden T, et al. Critical remarks on the use of terrestrial moss (Hylocomium splendens and Pleurozium schreberi) for monitoring of airborne pollution. Environ Pollut. 2001;113:41-57. DOI: 10.1016/S0269-7491(00)00156-1.

[24] Cempel M, Nikel G. Nickel: A review of its sources and environmental toxicology. Pol J Environ Stud. 2006;15(3):375-382. https://pdfs.semanticscholar.org/8a5b/14077da5c39096dff40d6177314538153b85.pdf.

[25] Garelick H, Jones H, Dybowska A, Valsami-Jones E. Arsenic pollution sources. Rev Environ Contam Toxicol. 2008;197:17-60. DOI: 10.1007/978-0-387-79284-2.

[26] Grigoratos T, Martini G. Brake wear particle emissions: a review. Environ Sci Pollut Res Int. 2015;22:2491-2504. DOI: 10.1007/s11356-014-3696-8. 